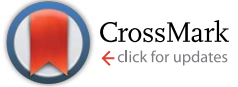

Cite this: J. Mater. Chem. A, 2017, 5, 3249

Received 4th December 2016 Accepted 13th January 2017

DOI: $10.1039 /$ c6ta10420g

www.rsc.org/MaterialsA

\section{The impact of lone-pair electrons on the lattice thermal conductivity of the thermoelectric compound $\mathrm{CuSbS}_{2}$}

\begin{abstract}
Baoli Du, ${ }^{\text {ab }}$ Ruizhi Zhang, ${ }^{\mathrm{b}}$ Kan Chen, ${ }^{\mathrm{b}}$ Amit Mahajan ${ }^{\mathrm{b}}$ and Mike J. Reece*b
The discovery and design of compounds with intrinsically low thermal conductivity, especially compounds with a special bonding nature and stable crystal structure, is a new direction to broaden the scope of potential thermoelectric (TE) materials. This study revealed unambiguously the origin of the impact of the lone pair electrons on lattice thermal conductivity in $\mathrm{Cu}-\mathrm{Sb}-\mathrm{S}$ compounds by correlating the special bonding on the $\mathrm{Sb}$ site with the phonon dispersion spectrum and density of states. By substitution of $\mathrm{Sb}$ with the transition metal Fe and group $I I_{A}$ element Ga without $s^{2}$ electrons, lone-pair electrons on some of the $\mathrm{Sb}$ sites were removed, which created a scenario with opposite influences on lattice thermal conductivity from the loss of lone-pair electrons and gain of alloy scattering. We investigated the competition between the alloy phonon scattering and the extra phonon scattering mechanism linked to lone-pair electrons on trivalent $\mathrm{Sb}^{3+}$ sites in chalcostibite $\mathrm{CuSbS}_{2}$, which is a model system for benchmarking and quantifying the impact of lone-pair electrons on the lattice thermal conductivity of $\mathrm{Cu}-\mathrm{Sb}-\mathrm{S}$ compounds. A significant deviation from the classic alloy model was observed. Along with the impact of the lone-pair electrons on the bonding arrangement and crystal structure, the role of lonepair electrons in the phonon transport of the TE compound $\mathrm{CuSbS}_{2}$ was well demonstrated and quantified. Two Sb-related quasi-single-frequency vibration modes behaving like localised Einstein harmonic oscillators were discovered and correlated with the bonding circumstance around $\mathrm{Sb}$ sites. These results give unequivocal evidence that the trivalent $V_{A}$ atom creates special bonding and vibration modes because of its nonbonding 5 s lone-pair electrons.
\end{abstract}

\section{Introduction}

The ability to discover and design materials with low lattice thermal conductivity is technologically important for thermoelectric (TE) generators and Peltier coolers, ${ }^{\mathbf{1 , 2}}$ which demand TE materials with crystal-like electrical transport properties and glass-like thermal conductivity, an ideal concept (phonon glasselectron crystal, PGEC) coined by Slack. ${ }^{3}$ Several strategies have been explored over the last decade to minimize the thermal conductivity of TE materials while delicately avoiding any detrimental effects on electrical properties. Benefiting from the difference between the phonon and charge carrier mean-free paths, ${ }^{4,5}$ introducing a high density of grain boundaries by embedding nanoscale 'guest domains' or nanopores in a 'host matrix' is an effective way to block/scatter the movement of phonons without serious degradation of electrical conductivity. A series of record high TE figure-of-merits, $z T$, were achieved in $\mathrm{AgPb}_{m} \mathrm{SbTe}_{2+m},{ }^{6} \mathrm{BiSbTe},{ }^{7,8}$ nano-microporous $\mathrm{AgSbTe}_{2}$ (ref. 9),

${ }^{a}$ School of Physics and Electronic Information Engineering, Henan Polytechnic University, Jiaozuo 454000, China.E-mail: dbl@hpu.edu.cn

${ }^{b}$ School of Engineering and Materials Science, Queen Mary University of London, London E1 4NS, UK. E-mail:m.j.reece@qmul.ac.uk skutterudites ${ }^{10}$ and other systems by this method. ${ }^{11,12}$ Another strategy is to use the 'crystal complexity' to decrease the thermal conductivity and enhance the electrical properties. ${ }^{13}$ Some quantitative discussion has been carried out for the complex disordered system $\mathrm{YbB}_{44} \mathrm{Si}_{2}$ (ref. 14). For layered materials, it is an effective way to optimise the TE properties by decoupling the interrelated thermal and electrical transport parameters by designing a complex crystal structure with one block exhibiting excellent electrical properties, and another block acting as a phonon scatterer to minimize the thermal transport. Layered materials, including $\mathrm{Na}-\mathrm{Co}-\mathrm{O},{ }^{15} \mathrm{TiS}_{2}($ ref. 16$)$ and $\mathrm{Bi}-\mathrm{Cu}-\mathrm{O}-\mathrm{Se}^{17}$ are representative examples of this design strategy. Inserting/ filling rattling atoms into oversized cages/voids is another proven promising method to obtain the ideal phonon-glass thermal conductivity, while still maintaining electron-crystal electrical transport properties along the crystalline frame sublattice or cages. ${ }^{18,19}$ The vibration of the rattler strongly couples to the frame/cage vibration modes, which lowers the velocity of the phonons and results in low thermal conductivity. ${ }^{\mathbf{2 0 2 1}}$ This strategy was well demonstrated both in skutterudites and clathrates. $^{22,23}$

Recently, Li et al. $^{24}$ discovered the link between lattice vibration anharmonicity and electronic orbitals in $\mathrm{SnSe}^{25}$ and 
revived the idea of designing materials with large Grüneisen parameters by anharmonicity engineering as proposed by Händel. ${ }^{26}$ Heremans $^{27}$ pointed out that high anharmonicity mostly occurs under conditions that are very close to a collapse of the crystal structure itself, which is verified by the unstable electrical structure and the ferroelectric-like lattice instability aroused by orbital interactions in SnSe. ${ }^{24}$ Another extreme example supporting Heremans's theory is copper selenide where Se atoms form a rigid cubic lattice with superionic copper ions with liquid-like mobility around them. ${ }^{28}$ The extraordinary 'liquid-like' nature of copper ions decreases the number of phonon modes and results in an intrinsically very low thermal conductivity in $\mathrm{Cu}_{2-x} \mathrm{Se}$ (around or less than $1 \mathrm{Wm}^{-1} \mathrm{~K}^{-1}$ for $\left.\mathrm{Cu}_{2} \mathrm{Se}\right){ }^{28,29}$ So, the discovery and design of compounds with intrinsically low thermal conductivity is a new direction to broaden the scope of potential TE materials, especially compounds with a special bonding nature and stable crystal structure.

In 2011, Skoug and Morelli ${ }^{30}$ investigated the thermal transport properties of the $\mathrm{Cu}-\mathrm{Sb}-\mathrm{Se}$ (CASe) system and found that the $\mathrm{Cu}_{3} \mathrm{SbSe}_{3}$ compound exhibits an abnormally low and nearly temperature-independent lattice thermal conductivity, whereas the structurally related $\mathrm{Cu}_{3} \mathrm{SbSe}_{4}$ does not show the same behaviour. ${ }^{31-33}$ They proposed that the electrostatic repulsion between the $5 \mathrm{~s}^{2}$ orbital lone-pair electrons on the trivalent $\mathrm{Sb}$ atoms and neighbouring chalcogenide ions results in soft phonon modes and strong vibrational anharmonicity, which in turn arouse the ultralow thermal conductivity of $\mathrm{Cu}_{3}-$ $\mathrm{SbSe}_{3}$ compound. ${ }^{30,34}$ This concept has been further validated by first-principles density-functional theory calculations of several group $\mathrm{I}_{\mathrm{B}}-\mathrm{V}_{\mathrm{A}}-\left(\mathrm{VI}_{\mathrm{A}}\right)_{2}$ and other compounds. ${ }^{35-38}$ The compounds always show lattice instabilities or different structures to those of the starting rocksalt structure, which result in strong phonon-phonon interactions and ultralow thermal conductivity approaching the amorphous limit. ${ }^{35}$ In fact, seminal work in 2008 reported $\mathrm{I}_{\mathrm{B}}-\mathrm{V}_{\mathrm{A}}-\left(\mathrm{VI}_{\mathrm{A}}\right)_{2}$ compounds with abnormally high Grüneisen parameters and soft frequency lattice vibration modes, for example AgSbTe ${ }_{2}$ and $\mathrm{AgBiSe}_{2}$ (ref. 34). The phonon mean free path is restricted to the interatomic distance by intrinsic normal and Umklapp phonon-phonon scattering processes alone because of the strong anharmonicity related to the special bonding arrangement. This has motivated research interest in the effect of bonding around trivalent group $\mathrm{V}_{\mathrm{A}}$ atoms on lattice thermal conductivity in related $\mathrm{I}_{\mathrm{B}}-\mathrm{V}_{\mathrm{A}}-\mathrm{VI}_{\mathrm{A}}$ compounds, containing group $\mathrm{I}_{\mathrm{B}}(\mathrm{Cu}, \mathrm{Ag})$, group $\mathrm{V}_{\mathrm{A}}(\mathrm{P}, \mathrm{As}, \mathrm{Sb}$, $\mathrm{Bi})$, and group $\mathrm{VI}_{\mathrm{A}}(\mathrm{S}, \mathrm{Se}, \mathrm{Te})$ elements. ${ }^{39,40}$ Most of the research focused on elucidating the relationship between the bonding arrangement and the low thermal conductivity by comparison of materials incorporating nominally trivalent $\mathrm{VI}_{\mathrm{A}}$ elements with materials incorporating only $\mathrm{V}_{\mathrm{A}}$ or $\mathrm{III}_{\mathrm{A}}$ elements. . $^{30,34,41}$ Actually, there is another way to assess the impact of the lonepair electrons on thermal conductivity by partly substituting the $\mathrm{V}_{\mathrm{A}}$ atoms by $\mathrm{III}_{\mathrm{A}}$ atoms without lone-pair electrons. First, this operation would introduce extra point defect phonon scattering and reduce the thermal conductivity based on the alloying model. Simultaneously, the substitution would break the special bonding arrangement related to the lone-pair electrons on some of the trivalent $\mathrm{V}_{\mathrm{A}}$ atom sites, which would likely restore the thermal conductivity to a typical value for a $\mathrm{I}_{B}{ }^{-}$ $\mathrm{III}_{\mathrm{A}}-\mathrm{VI}_{\mathrm{A}}$ compound. Since both effects happen on the atomic scale, it is reasonable to evaluate the role of the lone-pair electrons by analysing the doping concentration dependence of thermal conductivity as long as a system is free from the influences of any other factors.

$\mathrm{AgSbTe}_{2}$ is a representative example of $\mathrm{I}_{\mathrm{B}}-\mathrm{V}_{\mathrm{A}}-\left(\mathrm{VI}_{\mathrm{A}}\right)_{2}$ group compounds, and is a well-studied system with intrinsic minimum thermal conductivity $\left(<0.6-0.7 \mathrm{Wm}^{-1} \mathrm{~K}^{-1}\right) \cdot{ }^{9,34-36,42,43}$ However, its 'cubic' crystal structure is still a controversial issue. ${ }^{36}$ Also, the spontaneously generated nanostructure produced by a natural formation of nanoscale domains with different ordering on the cation sublattice plays some role in scattering phonons and achieving a low thermal conductivity. ${ }^{44}$ Bismuth copper oxychalcogenides BiOCuQ $(\mathrm{Q}=\mathrm{Se}, \mathrm{Te})$ are another hot topic related to the impact of lone-pair electrons on thermal conductivity. ${ }^{45,46}$ Recently, first-principles calculations and in situ neutron diffraction analysis have suggested that the low thermal conductivity of those materials may be attributed to the weak bonding of copper atoms within the structure, rather than to the $\mathrm{Bi}^{3+}$ lone pairs. ${ }^{47}$ There is a sulphide counterpart (Cu-Sb-S, CAS system) of the CASe system..$^{39}$ The four main crystalline phases of the CAS compounds are $\mathrm{Cu}_{3} \mathrm{SbS}_{4}$ (famatinite), ${ }^{48,49} \mathrm{Cu}_{3} \mathrm{SbS}_{3.25}$ (tetrahedrite, $\mathrm{Cu}_{12} \mathrm{Sb}_{4} \mathrm{~S}_{13}$ ), ${ }^{50-52} \mathrm{Cu}_{3} \mathrm{SbS}_{3}$ (skinnerite), ${ }^{53}$ and $\mathrm{CuSbS}_{2}$ (chalcostibite). ${ }^{54-56}$ Fig. 1 shows the temperature dependence of the lattice thermal conductivity of all of the four compounds. ${ }^{41,57}$ Famatinite has the highest value in nearly the whole temperature range, while tetrahedrite and skinnerite exhibit abnormally low and nearly temperatureindependent lattice thermal conductivity. Fig. 2 shows the crystal structures of the members of the CAS system. The $\mathrm{Sb}$ atoms in famatinite are tetrahedrally coordinated with four $\mathrm{S}$ atoms by $\mathrm{sp}^{3}$ hybridisation..$^{58}$ No non-bonded $\mathrm{Sb} 5 \mathrm{~s}^{2}$ lone-pair elections exist, which results in a relatively high lattice thermal conductivity. In contrast, the other three compounds

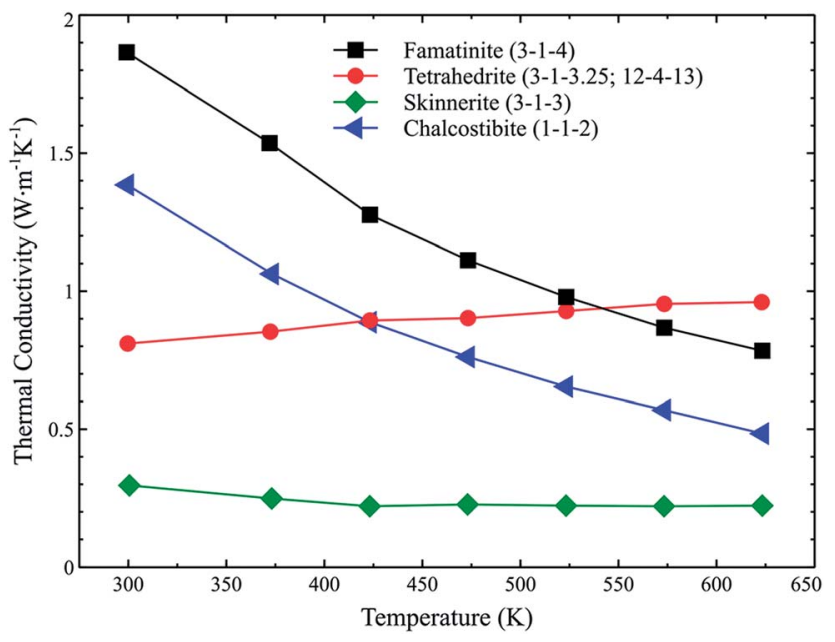

Fig. 1 Temperature dependence of the lattice thermal conductivity of copper antimony sulphide, including famatinite, tetrahedrite, skinnerite, and chalcostibite. ${ }^{41,57}$ 
(a) Famatinite

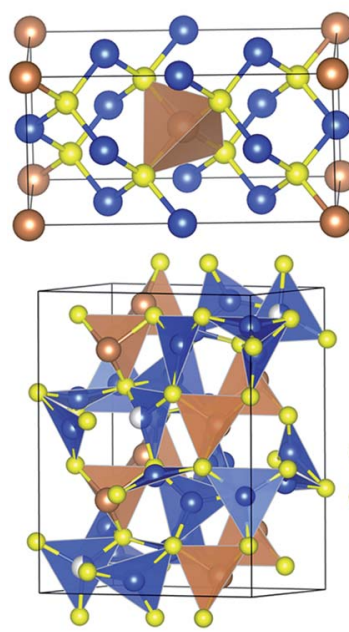

(c) Skinnerite (b) Tetrahedrite
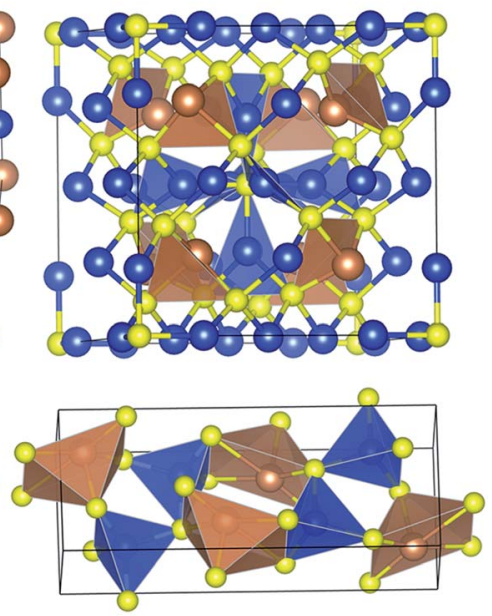

(d) Chalcostibite
Fig. 2 Crystal structures of (a) famatinite (3-1-4), (b) tetrahedrite (3-1-3.25 or 12-4-13), (c) skinnerite (3-1-3) and (d) chalcostibite (1-1-2). The small balls represent $\mathrm{S}$ (yellow), medium size $\mathrm{Cu}$ (blue) and large ones indicate $\mathrm{Sb}$ atoms (brown). All the structures were created using Vesta software.

have spare $\mathrm{Sb} 5 \mathrm{~s}^{2}$ electrons free to orient along the missing vertex of their associated polyhedron. Tetrahedrite, which does not have a counterpart in the CASe system, forms a cubic structure, where half of the copper atoms occupy three-fold coordinated $\mathrm{CuS}_{3}$ trigonal sites. The Sb lone-pair electrons on both sides of the $\mathrm{CuS}_{3}$ triangular plane weaken the bonding of the $\mathrm{Cu}$ atoms in the direction perpendicular to the $\mathrm{CuS}_{3}$ plane, which leads to quasi-localised and anharmonic out-of-plane rattling modes. This is likely to be the origin of the low thermal conductivity of tetrahedrite. ${ }^{30,59}$ There are no less than three temperature-dependent polymorphs of $\mathrm{Cu}_{3} \mathrm{SbS}_{3}$ (ref. 53). All of the structures show mixed character with lone-pair electron bonding arrangement and $\mathrm{CuS}_{3}$ trigonal bonding units or fractionally occupied $\mathrm{Cu}$ sites, including the room temperature polymorph skinnerite. For similar reasons, both tetrahedrite and skinnerite are more thermally insulating than famatinite at room temperature and have nearly constant thermal conductivity with increasing temperature. So, both tetrahedrite and $\mathrm{Cu}_{3} \mathrm{SbS}_{3}$ are not ideal compounds to evaluate the effect of lonepair electrons on thermal conductivity because of the influences of other factors. Chalcostibite forms a stable orthorhombic structure (space group Pnma) until its melting point. ${ }^{60}$ It has lower thermal conductivity than famatinite but exhibits a similar trend, with intrinsic Umklapp phonon scattering dominating its thermal conductivity behaviour. All the $\mathrm{Cu}$ atoms are tetrahedrally coordinated in $\mathrm{CuS}_{4}$ units. The edgeshared square pyramidal $\mathrm{SbS}_{5}$ units are separated by $\mathrm{CuS}_{4}$ units and face one another, which directs the lone-pair electrons into the void separating the $\mathrm{SbS}_{5}$ units. In contrast to the co-contributions from mixed factors in tetrahedrite and skinnerite, the lone-pair electrons are the solo possible factor accounting for the low thermal conductivity in chalcostibite. So, chalcostibite is an ideal system to assess the role of lone-pair electrons free from the influences of other factors.
To clarify the importance of lone-pair electrons on trivalent $\mathrm{Sb}^{3+}$ sites in $\mathrm{CuSbS}_{2}$ compound, alloy phonon scattering was purposely introduced and set up as a benchmark to quantify the impact of lone-pair electrons on thermal conductivity. By substitution of $\mathrm{Sb}$ with the transition metal $\mathrm{Fe}$ and group $\mathrm{III}_{\mathrm{A}}$ element $\mathrm{Ga}$, lone-pair electrons on a fraction of the $\mathrm{Sb}$ sites were removed. All of the outer shell electrons around the Fe (Ga) sites involve in the formation of $\mathrm{sp}^{3}$ hybridisation due to the valence number difference between $\mathrm{Sb}$ and $\mathrm{Fe}(\mathrm{Ga})$. We expected that there would be a deviation from the alloy mode of thermal conductivity in a system with opposite influences of the loss of lone-pair electrons and gain of point defects. In this study, the competing impacts of lone-pair electrons and point defects on thermal conductivity were analysed. The doping concentration dependent thermal conductivity and phonon spectrum were also studied and correlated.

\section{Experimental details}

Two alloy systems $\mathrm{CuFe}_{x} \mathrm{Sb}_{1-x} \mathrm{~S}_{2}$ and $\mathrm{CuGa}_{x} \mathrm{Sb}_{1-x} \mathrm{~S}_{2}(x=0,0.01$, $0.025,0.05,0.1,0.2,0.9$, and 1 ) were designed to remove lonepair electrons from some of the $\mathrm{Sb}$ sites by incorporating the trivalent transition metal atom $\mathrm{Fe}$ and group $\mathrm{III}_{\mathrm{A}}$ atom $\mathrm{Ga}$ in chalcostibite. Both systems were synthesised using a mechanical-alloying spark-plasma-sintering synthesis route. Pure elements $\mathrm{Cu}$ (150 mesh, 99.5\%), Sb (100 mesh, 99.5\%), S (reagent grade, purified by sublimation, $100 \mathrm{mesh}$ ) and $\mathrm{Fe}(200$ mesh, 99+\%) were used as raw materials to synthesise $\mathrm{CuFe}_{x^{-}}$ $\mathrm{Sb}_{1-x} \mathrm{~S}_{2}$, while $\mathrm{Ga}_{2} \mathrm{~S}_{3}$ (powder, $99.99 \%$, metals basis) was used as the gallium source to synthesise $\mathrm{CuGa}_{x} \mathrm{Sb}_{1-x} \mathrm{~S}_{2}$. The powders were weighed and then sealed in stainless steel milling jars in an Ar filled glovebox. The jars were mounted and milled in a planetary mill at a rotational speed of $450 \mathrm{rpm}$ for $20 \mathrm{~h}$. The samples with $x \leq 0.2$ were spark plasma sintered using a graphite die in vacuum at $400^{\circ} \mathrm{C}$ for $5 \mathrm{~min}$. For samples with $x$ $=0.9$ or 1 , the sintering temperature was adjusted based on the melting point of $\mathrm{CuFeS}_{2}\left(950^{\circ} \mathrm{C}\right)$ and $\mathrm{CuGaS}_{2}\left(1236^{\circ} \mathrm{C}\right)$ to achieve dense pellets. For comparison, $\mathrm{Cu}_{3} \mathrm{SbS}_{3}$ was synthesised using the same processing method and its thermal conductivity is listed in Fig. 1 with tetrahedrite, famatinite and chalcostibite.

The constituent phases of the samples were characterized using powder X-ray diffraction (XRD, X'Pert PRO-PANalytical, $\mathrm{CuK} \alpha$ ) in the range $5-120^{\circ}$. RAMAN spectra were obtained from powders using a RENISHAW machine equipped with a He-Ne laser source with $633 \mathrm{~nm}$ wavelength and optical lens of $50 \times$. The error associated with RAMAN measurements was 1 $\mathrm{cm}^{-1}$. The microstructure images of freshly fractured surfaces were observed using a scanning electron microscope (SEM, FEI Inspect TM-F) with energy dispersive X-ray spectroscopy (EDS). The temperature dependent electrical resistivity and Seebeck coefficient of $\mathrm{CuFe}_{x} \mathrm{Sb}_{1-x} \mathrm{~S}_{2}(x=0.9$, and 1$)$ were measured using a commercial instrument (ZEM-3, Ulvac, Inc.) in a $\mathrm{He}$ atmosphere. The error of resistivity and Seebeck coefficient measurements are less than $5 \%$. Thermal conductivity $\kappa$ was determined using the equation $\kappa=\lambda C_{\mathrm{p}} d$. The temperature dependent thermal diffusivity $\lambda$ was measured using a laser flash method (LFA-457, Netzsch) on a pellet ( $\phi 12.7 \mathrm{~mm}$, height 
$1.5 \mathrm{~mm}$ ). The machine was calibrated using a standard specimen within the same temperature range. The repeatability of the measurement was better than $2 \%$, while the error of the thermal diffusivity was less than $5 \%$. The specific heat $C_{\mathrm{p}}$ was calculated using the Dulong-Petit law to avoid the large uncertainty in the routine differential scanning calorimetry method. The density $d$ was obtained using the mass and volume of the sintered pellets with an error less than $1 \%$.

\section{Computational details}

First principles calculations were performed using the Quantum-ESPRESSO package. ${ }^{61}$ We used the Garrity-BennettRabe-Vanderbilt (GBRV) high-throughput pseudopotential library. ${ }^{62}$ The Perdew-Burke-Ernzerhof (PBE) function was used along with ultrasoft pseudopotentials for all the atoms. A plane wave basis with kinetic energy cut off of at $500 \mathrm{eV}$ and a dense $k$ point sampling of $7 \times 11 \times 3$ were used to ensure the convergence in all of the calculations. The atomic positions were relaxed until all the force components on each atom were less than $10^{-3}$ a.u., and the lattice constants were optimized until the stress was less than $0.5 \mathrm{kbar}$. The phonon dispersions and partial phonon density of states were calculated using the density perturbation functional theory (DPFT) and QuasiHarmonic Approximation (QHA) package, both implemented in Quantum-ESPRESSO.

\section{Results and discussion}

According to the literature and the above structural analysis, the lone-pair electrons are probably the solo decisive factor in determining the ultralow thermal conductivity of single crystal chalcostibite. To discuss the competing impacts of the loss of lone-pair electrons and the gain of point defects in the substituted polycrystalline samples, we need to separate their effect from other influences, such as phase structure and microstructure.

\section{Phase structure}

Fig. 3 shows the X-ray diffraction spectra of (a) $\mathrm{CuGa}_{x} \mathrm{Sb}_{1-x} \mathrm{~S}_{2}(x$ $=0,0.01,0.025,0.05,0.1,0.2,0.9$, and 1) and (b) $\mathrm{CuFe}_{x} \mathrm{Sb}_{1-x} \mathrm{~S}_{2}$ $(x=0,0.01,0.025,0.05,0.1,0.2,0.9$, and 1$)$. The two bottom traces were generated based on the crystal structures of pure $\mathrm{CuSbS}_{2}$ and $\mathrm{CuGaS}_{2} / \mathrm{CuFeS}_{2}$. For both the Fe and Ga substituted samples, the samples are divided into two groups based on the crystal structure. Samples with $x$ up to 0.2 crystallise with the chalcostibite structure without any trace of impurity. Samples with $x=0.9$ and 1 are phase-pure materials with a chalcopyrite structure (cation ordered structure based on zinc blende) for both Ga and Fe substituted samples. This confirmed that it is possible to maintain the structure of chalcostibite even when $20 \%$ of the lone-pair electrons are removed from the trivalent $\mathrm{Sb}$ sites. Based on the principle of minimum energy, the $\mathrm{Fe} / \mathrm{Ga}$ atoms should be randomly distributed on the $\mathrm{Sb}$ sites of chalcostibite, which in turn restrains the formation of a secondary phase. However, the chalcostibite structure collapses without the support from the lone-pair electrons in the voids separating the $\mathrm{SbS}_{5}$ units in samples with a specific value of $x$ between 0.2 and 0.9. The phases of $\mathrm{CuFeS}_{2}$ and $\mathrm{CuGaS}_{2}$ with the chalcopyrite structure then form with tetrahedral coordination geometry similar to famatinite.

\section{Raman spectroscopy analysis}

To clarify the effect of Ga doping on the structure, the Ramanactive modes were investigated at ambient temperature for $\mathrm{CuSbS}_{2}, \mathrm{CuGa}_{0.025} \mathrm{Sb}_{0.975} \mathrm{~S}_{2}$ and $\mathrm{CuGaS}_{2}$ (Fig. 4). The $\mathrm{CuSbS}_{2}$ sample has three pronounced peaks at about 187, 250 and 329 $\mathrm{cm}^{-1}$, along with a much weaker peak at about $450 \mathrm{~cm}^{-1}$, which is in agreement with data reported in the literature.$^{63}$ The broad peak at $329 \mathrm{~cm}^{-1}$ includes two adjoining modes with $A_{\mathrm{g}}$ symmetry, which are assigned to the vibration modes from the
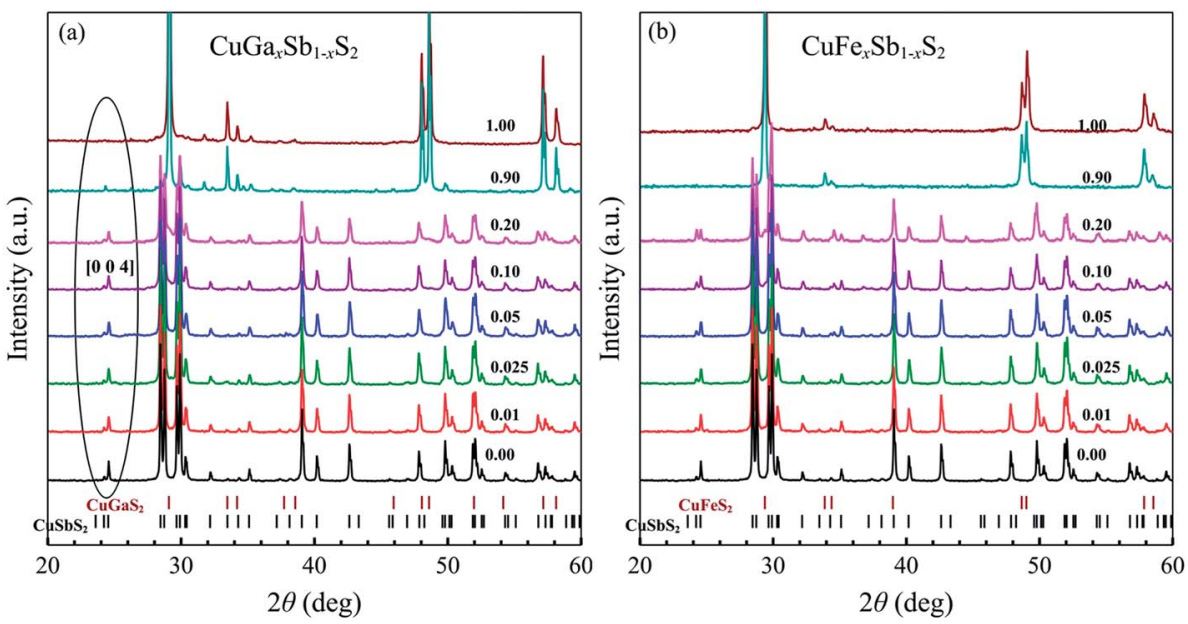

Fig. $3 X$-ray diffraction spectra of (a) CuGa $\mathrm{Sb}_{1-x} \mathrm{~S}_{2}(x=0,0.01,0.025,0.05,0.1,0.2,0.9$, and 1$)$ and (b) $\mathrm{CuFe}_{x} \mathrm{Sb}_{1-x} \mathrm{~S}_{2}(x=0,0.01,0.025,0.05$, $0.1,0.2,0.9$, and 1). The peaks related to the $\left[\begin{array}{ll}0 & 0\end{array}\right]$ plane are labeled in (a). The two bottom traces were generated for pure $\mathrm{CuSbS}_{2}$ and $\mathrm{CuGaS}_{2} /$ $\mathrm{CuFeS}_{2}$. The samples are categorized into two groups based on the crystal structure in both Ga and Fe doped systems. 


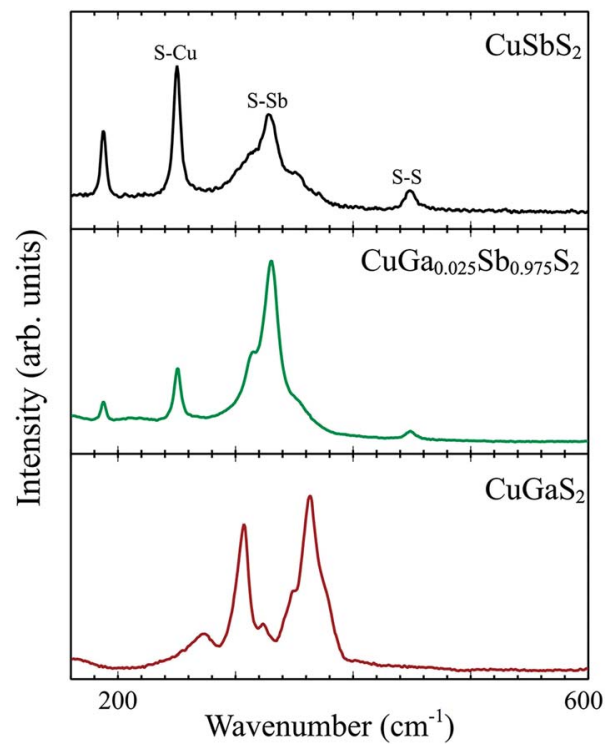

Fig. 4 Raman spectra of (a) $\mathrm{CuSbS}_{2}$, (b) $\mathrm{CuGa}_{0.025} \mathrm{Sb}_{0.975} \mathrm{~S}_{2}$ and (c) $\mathrm{CuGaS}_{2}$ samples.

$\mathrm{Sb}-\mathrm{S}$ bonds. Peaks at 250 and $450 \mathrm{~cm}^{-1}$ are attributed to the vibration of $\mathrm{Cu}-\mathrm{S}$ and $\mathrm{S}-\mathrm{S}$ bonds, respectively. Compared to $\mathrm{CuSbS}_{2}$ the peak positions of $\mathrm{CuGa}_{0.025} \mathrm{Sb}_{0.975} \mathrm{~S}_{2}$ are not shifted, but there is a significant change in the relative peak intensities. This probably means that $2.5 \mathrm{~mol} \%$ of Ga doping shifts the orientation of the $\mathrm{Sb}-\mathrm{S}$ bonds, which also manifested itself in the XRD pattern. As shown in Fig. 3(a), the intensity of the [ll 004$]$ peak at $24.5^{\circ}(2 \theta)$ decreases with increasing Ga substitution. In pristine $\mathrm{CuSbS}_{2}$, two $\mathrm{S}$ atoms of the bottom face of one pyramidal $\mathrm{CuS}_{5}$ and the top $\mathrm{S}$ atom of the opposite $\mathrm{CuS}_{5}$ unit form the $\left[\begin{array}{lll}0 & 0 & 4\end{array}\right]$ atomic plane. Any doping on the $\mathrm{Sb}$ sites with atoms without lone-pair electrons causes a delicate reconstruction of the $\mathrm{CuS}_{5}$ unit, which leaves a ragged [ $\left[\begin{array}{lll}0 & 0 & 4\end{array}\right]$ lattice plane, which in turn decreases the XRD intensity from the plane. In moving from $\mathrm{CuSbS}_{2}$ to $\mathrm{CuGaS}_{2}$, the peak at $329 \mathrm{~cm}^{-1}$ disappears completely and an intense peak at $307 \mathrm{~cm}^{-1}$ emerges due to the $\mathrm{A}_{1}$ vibrational mode in the chalcopyrite structure. The above discussion showed that the special bonding circumstance on $\mathrm{Sb}$ sites in chalcostibite plays an important role in determining its crystal structure.

\section{Microstructure}

Fig. 5 shows the typical SEM images of the $\mathrm{Cu}(\mathrm{Fe} / \mathrm{Ga})_{x} \mathrm{Sb}_{1-x} \mathrm{~S}_{2}$ samples. All samples are free of pores and have relative densities between $98 \%$ and $99 \%$. Due to the different crystalline structures and sintering temperatures, samples with $x$ up to 0.2 have very fine grain size ranging from 100 to $300 \mathrm{~nm}$ as shown in Fig. 5(a), while samples with the chalcopyrite structure $(x=$ 0.9 and 1) have coarse grains between 1 and $4 \mu \mathrm{m}$ as shown in Fig. 5(b). According to the phase structure and microstructure, we separated the samples into two categories. One is the samples with $x$ up to 0.2 sharing the chalcostibite structure and fine average grain size, the other is the samples with $x=0.9$ and 1 with tetrahedral coordination geometry and coarse grains.

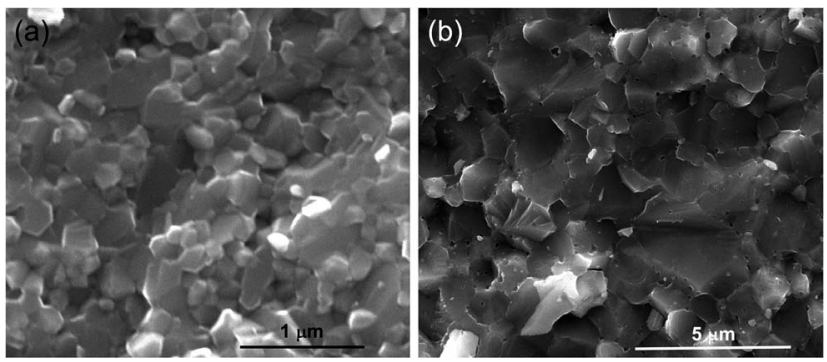

Fig. 5 Typical SEM images of bulk $\mathrm{Cu}(\mathrm{Fe} / \mathrm{Ga})_{x} \mathrm{Sb}_{1-x} \mathrm{~S}_{2}$ samples with (a) $x$ up to 0.2 and (b) $x=0.9$ or 1 .

This classification made it possible to put aside the influences of phase and microstructure on thermal conductivity in the following discussion.

\section{Thermal conductivity and TE properties of the $\mathrm{CuFe}_{x} \mathrm{Sb}_{1-x} \mathrm{~S}_{2}$ system}

Fig. 6 shows the temperature dependence of thermal conductivity $\kappa$ for $\mathrm{CuFe}_{x} \mathrm{Sb}_{1-x} \mathrm{~S}_{2}(x=0,0.01,0.025,0.05,0.1,0.2,0.9$, and 1). The sample category with $x$ up to 0.2 possesses much lower thermal conductivity than the other category with $x=0.9$ and 1. The thermal conductivities decrease with increasing temperature for all of the samples, which indicates that Umklapp phonon scattering prevails in the testing temperature range. No apparent ambipolar transport phenomenon was observed. The overall thermal conductivity is the sum of two nearly independent terms $\kappa=\kappa_{\text {latt }}+\kappa_{\text {carr }}$, where $\kappa_{\text {latt }}$ and $\kappa_{\text {carr }}$ are the lattice and carrier contributions, respectively. The carrier term is related to the electrical resistivity $\rho$ via the WiedemannFranz law, $\kappa_{\text {carr }}=L T / \rho$, where $L$ is the Lorentz constant, which is $2.45 \times 10^{-8} \mathrm{~V}^{2} \mathrm{~K}^{-2}$ for a fully degenerate semiconductor. ${ }^{64}$ The high resistivity of the samples with $x$ up to 0.2 means that their carrier thermal conductivity accounts for less than $0.1 \%$ of their overall thermal conductivity. However, the carrier contributions

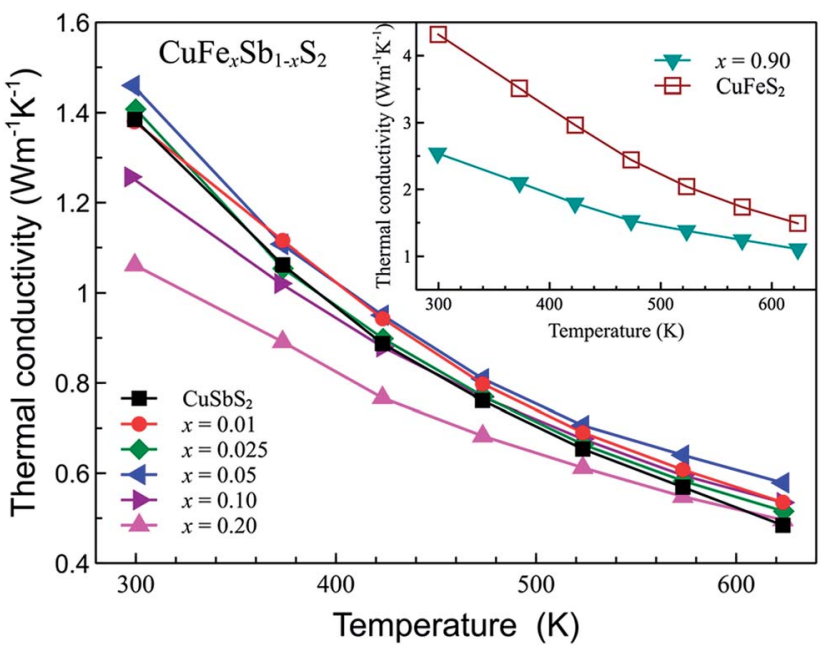

Fig. 6 Temperature dependence of thermal conductivity of $\mathrm{CuFe}_{x}-$ $\mathrm{Sb}_{1-x} \mathrm{~S}_{2}(x=0,0.01,0.025,0.05,0.1,0.2,0.9$, and 1$)$. 
in $\mathrm{CuFe}_{0.9} \mathrm{Sb}_{0.1} \mathrm{~S}_{2}$ and $\mathrm{CuFeS}_{2}$ are not negligible, and were calculated using the Wiedemann-Franz law. Fig. 7 displays the resistivity, Seebeck coefficient, lattice/carrier thermal conductivity and figure-of-merit of the $\mathrm{CuFe}_{0.9} \mathrm{Sb}_{0.1} \mathrm{~S}_{2}$ and $\mathrm{CuFeS}_{2}$ samples. Both samples have rather high Seebeck coefficient and moderate resistivity and thermal conductivity. A figure-of-merit $z T$ of 0.19 was obtained for the $\mathrm{CuFe}_{0.9} \mathrm{Sb}_{0.1} \mathrm{~S}_{2}$ sample. This value is comparable to values reported in the literature, ${ }^{65,66}$ which demonstrates that n-type $\mathrm{CuFeS}_{2}$ is a good candidate to work with the $p$-type members of the CAS system in TE generators in the intermediate temperature range.

\section{Lattice thermal conductivity}

Fig. 8 displays the temperature dependence of lattice thermal conductivity for (a) $\mathrm{CuFe}_{x} \mathrm{Sb}_{1-x} \mathrm{~S}_{2}(x=0,0.01,0.025,0.05,0.1$, 0.2, 0.9, and 1) and (b) $\mathrm{CuGa}_{x} \mathrm{Sb}_{1-x} \mathrm{~S}_{2}(x=0,0.01,0.025,0.05$, $0.1,0.2,0.9$ and 1$)$. The carrier thermal conductivity for all of the Ga substituted samples is negligible, including the $\mathrm{CuGaS}_{2}$ sample due to their high resistivity. ${ }^{67}$ So, we regarded the total thermal conductivity of all the Ga substituted samples as their lattice thermal conductivity in the following discussion. The $\mathrm{CuGa}_{x} \mathrm{Sb}_{1-x} \mathrm{~S}_{2}$ system is also divided into two categories based on their crystal structure and microstructure, as was done for the $\mathrm{CuFe}_{x} \mathrm{Sb}_{1-x} \mathrm{~S}_{2}$ system. Excluding the $x=0.9$ and 1 samples with different crystal structures, the thermal conductivities initially increase slightly, reach a maximum, and then decrease with the increasing substitution in both systems. This trend apparently contradicts with the alloying model. In conventional solid solutions or alloys, point defect phonon scattering is one of the dominant factors in reducing the lattice thermal conductivity because of mass contrast, charge fluctuation, local strain and other accompanying changes around the foreign atoms. Historically, alloying is the cornerstone for the design of the most commercial room temperature TE materials (bismuth chalcogenide solid solutions) ${ }^{68}$ and high temperature materials (SiGe). ${ }^{69}$ To illustrate the results more clearly, the lattice thermal conductivities are re-plotted against substitution concentration at chosen temperatures, 300, 373 and $600 \mathrm{~K}$ in Fig. 9. A 5\% error bar was used for each point. In both Fe and Ga substituted systems and at all temperatures, the lattice thermal conductivity increases in the lightly doped samples. Taking into account the special bonding arrangements and the electron density distribution around the trivalent $\mathrm{Sb}$ sites in chalcostibite, the abnormal lattice thermal conductivity increment in lightly doped samples is naturally connected to the $5 \mathrm{~s}^{2}$ lone-pair electrons. To quantify the impact of the lone-pair electrons on lattice thermal conductivity, the modelled lattice thermal conductivities based on an alloy model are also plotted in Fig. 9. According to Klemens and Abeles's model, ${ }^{70,71}$ the lattice thermal conductivity $\kappa$ of a disordered alloy can be calculated from the $\kappa_{\mathrm{p}}$ value in the absence of point defects (pure $\mathrm{CuSbS}_{2}$ in this work),

$$
\begin{aligned}
\frac{\kappa}{\kappa_{\mathrm{p}}}= & {\left[1+\frac{5}{9} a\right]^{-1} } \\
& \times\left[\frac{\tan ^{-1} U}{U}+\frac{\left(1-\left(\tan ^{-1} U / U\right)\right)^{2}}{[(1+a) / 5 a] U^{4}-\frac{1}{3} U^{2}+1-\left(\tan ^{-1} U / U\right)}\right],
\end{aligned}
$$

where $a$ is the ratio of the relaxation times of three-phonon normal and Umklapp process, and $U$ is calculated by the equation
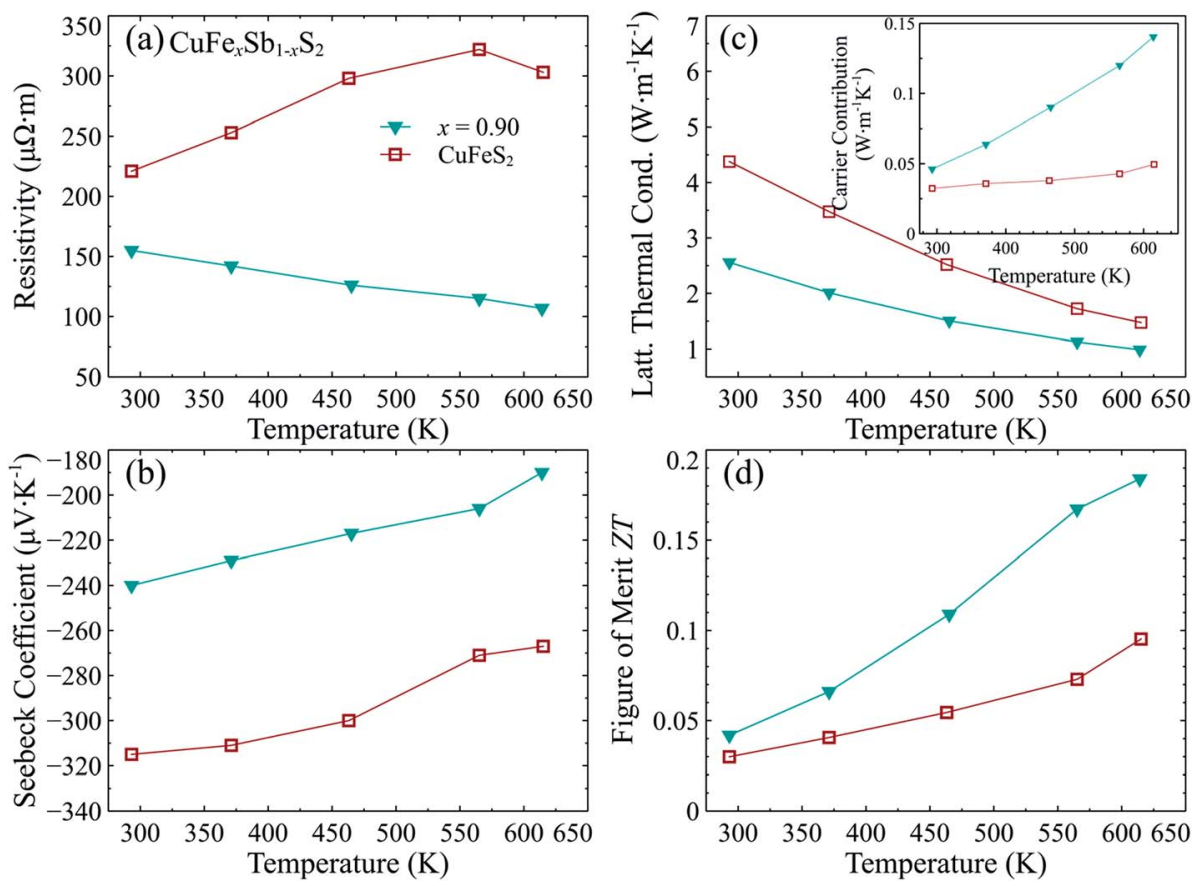

Fig. 7 Electrical, thermal transport properties, and figure-of-merit $z T$ of $\mathrm{CuFe}_{0.9} \mathrm{Sb}_{0.1} \mathrm{~S}_{2}$ and $\mathrm{CuFeS}_{2}$ samples. 

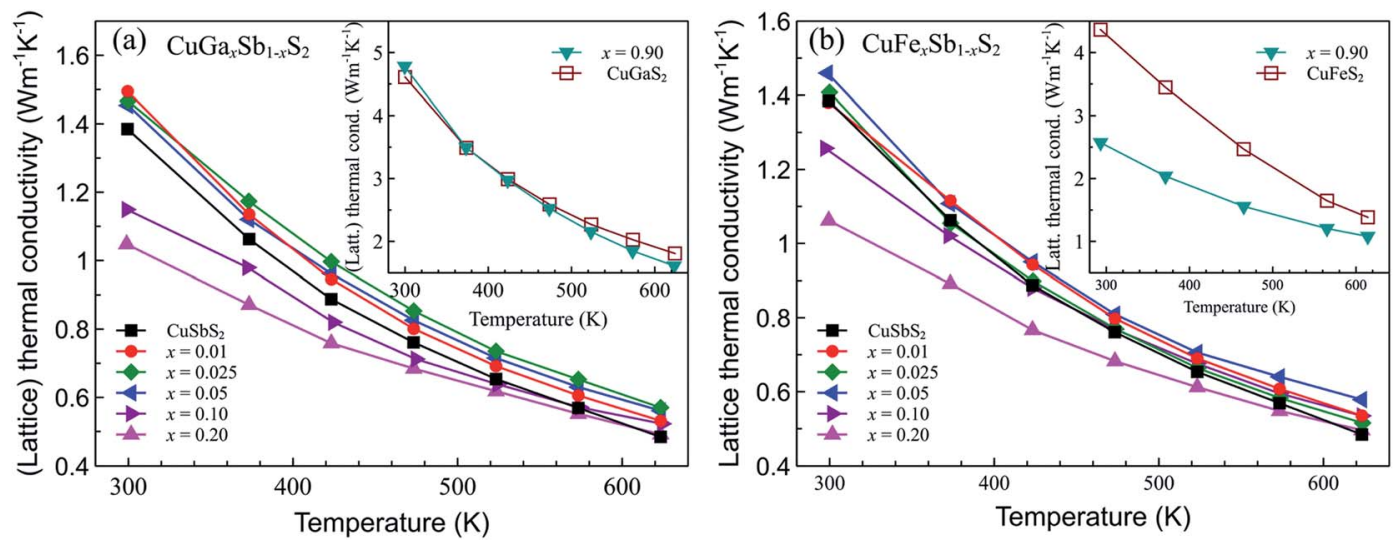

Fig. 8 Temperature dependence of lattice thermal conductivity of (a) $\mathrm{CuGa}_{x} \mathrm{Sb}_{1-x} \mathrm{~S}_{2}(x=0,0.01,0.025,0.05,0.1,0.2,0.9$, and 1$)$ and (b) $\mathrm{CuFe}_{x} \mathrm{Sb}_{1-x} \mathrm{~S}_{2}(x=0,0.01,0.025,0.05,0.1,0.2,0.9$ and 1$)$.

$$
U=8.69 \times 10^{6}\left(1+\frac{5}{9} a\right)^{1 / 2} \gamma^{-1} \beta \Gamma^{1 / 2} \delta^{-1 / 2} T^{-1 / 2}
$$

$\beta$ is nearly a constant within a given covalent crystal system. $\Gamma$ is a function of the strain parameter $\varepsilon$ and other known basic alloy parameters. $\delta^{3}$ is the atomic volume derived from Vegard's law. So, there are three adjustable parameters, the ratio of normal and Umklapp scattering rate $a$, the anharmonicity parameter $\gamma$, and the strain parameter $\varepsilon$, to estimate the thermal conductivities of alloys. As shown in Fig. 9, the discrepancy between the model values and the experimental data highlights the significance of the lone-pair electrons in chalcostibite. In alloy or solid solution, the lattice thermal conductivity is quite sensitive to the point defects due to its effective scattering of short and medium-wavelength heat-carrying phonons. However, the lattice thermal conductivity witnesses an increase rather than a decrease in lightly $\mathrm{Fe}$ and $\mathrm{Ga}$ doped chalcostibite. This suggests that the influence of the loss of lone-pair electrons outweighs the impact of point defects in lightly doped samples. With further increase in doping, the effect of phonon scattering by point defects becomes more dominant in the trend of the lattice thermal conductivity. This trend also manifested itself at $373 \mathrm{~K}$ and $600 \mathrm{~K}$, which indicates that lone-pair elections play an important role in thermal transport properties both at low and high temperature. Taking into account the origins of point defects scattering and lone-pair electrons, both mechanisms are closely related to the bonding properties at the atomic scale. So both phonon scattering mechanisms should have a similar working temperature range and compete with each other in the whole temperature range. Actually, the lattice thermal conductivity is controlled by the competition between the opposite effects of point defects and the loss of lone-pair electrons on $\mathrm{Sb}$ sites. This is the first direct demonstration of the role of lonepair electrons in comparison with another well-known scattering mechanism. It demonstrates that lone-pair electrons provide a strong mechanism to transform phonon transport and may eclipse point defects in lightly doped solid solution/ alloys. This suggests that compounds with lone-pair electrons (trivalent $\mathrm{V}_{\mathrm{A}}$ atoms) provide a new direction to design low thermal conductivity materials, and can be used to screen for prospective TE materials.
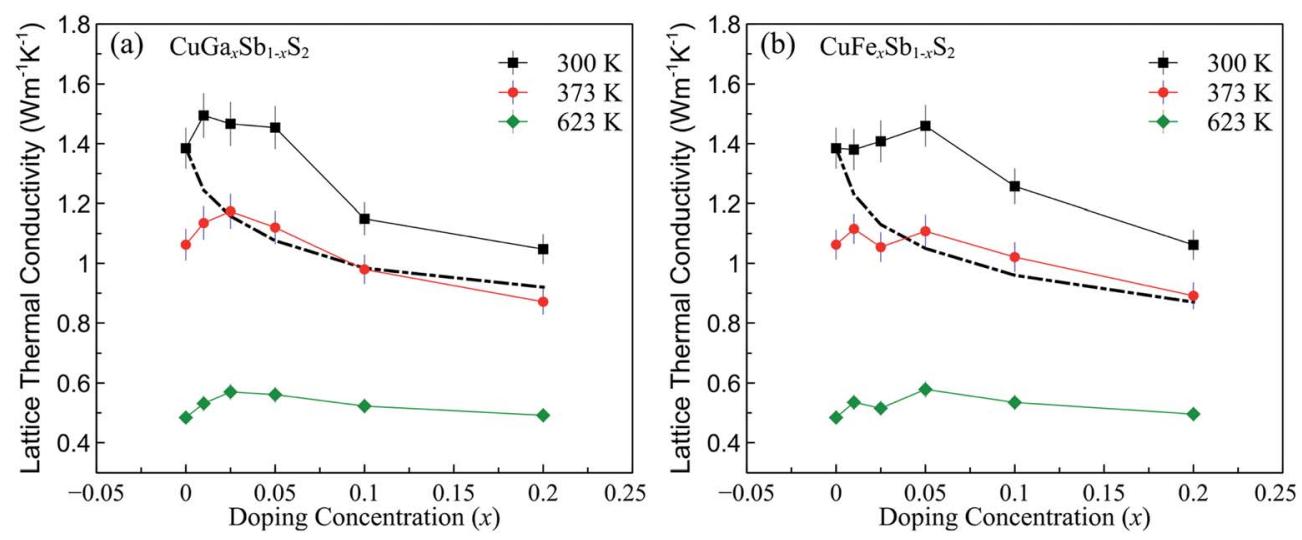

Fig. 9 Lattice thermal conductivity of (a) $\mathrm{CuGa}_{x} \mathrm{Sb}_{1-x} \mathrm{~S}_{2}(x=0,0.01,0.025,0.05,0.1,0.2,0.9$, and 1$)$ and (b) $\mathrm{CuFe}_{x} \mathrm{Sb}_{1-x} \mathrm{~S}_{2}(x=0,0.01,0.025,0.05$, 0.1 , and 0.2 ) at $300 \mathrm{~K}, 373 \mathrm{~K}$ and $600 \mathrm{~K}$. The dashed lines show the calculated lattice thermal conductivity of $\mathrm{CuGa}_{x} \mathrm{Sb}_{1-x} \mathrm{~S}_{2}$ and $\mathrm{CuFe}_{x} \mathrm{Sb}_{1-x} \mathrm{~S}_{2}$ based on the alloy model. ${ }^{70,71}$ 


\section{Phonon band structure and density of states}

To illustrate the mechanism of the effect of lone-pair elections on thermal transport properties, the phonon spectra of $\mathrm{CuSbS}_{2}$ and $\mathrm{CuGaS}_{2}$ were calculated (Fig. 10). Although the chalcostibite structure has been reported for $\mathrm{CuSbS}_{2}$ at room temperature, the negative phonon frequencies indicate that this structure is unstable at $0 \mathrm{~K}$, at which the first principles calculations were performed. The eigen-displacements of the negative modes (soft modes) will lead to phase transition at low temperatures, known as 'soft mode hardening'. In the following discussions, the phonon dispersion of the $\mathrm{CuSbS}_{2}$ chalcostibite structure will be used because the discussions focus on the lattice thermal conductivity above room temperature. Besides, most of the optical modes and the three acoustic modes are unaffected by the soft modes and have positive values.

Due to the high symmetry of the chalcopyrite structure, $\mathrm{CuGaS}_{2}$ has much fewer optical modes than $\mathrm{CuSbS}_{2}$. Most of the optical modes exhibit frequencies higher than $125 \mathrm{~cm}^{-1}$, except two modes at 92 and $94 \mathrm{~cm}^{-1}$. Compared to its Ga counterpart, the most obvious feature of $\mathrm{CuSbS}_{2}$ is that the frequencies of the optical modes are very low, although an underestimation of the frequencies may be possible because of the band-gap problem in generalized gradient approximation (GGA). In fact, most of the optical modes are located in a narrow low frequency range of $20-80 \mathrm{~cm}^{-1}$, which is similar to $\mathrm{AgSbTe}_{2}$. Ye et al. linked the softness of those modes to the heavy atomic masses, the relatively weak bonds between $\mathrm{Te}$ and $\mathrm{Sb}$, and the large anioncation distances. ${ }^{36}$ In chalcostibite, the $\mathrm{Sb} 5 \mathrm{~s}^{2}$ electrons orienting toward the voids between the $\mathrm{SbS}_{5}$ units are nonbonding, which may lead to a softening of the optical modes. The small gap between the optical modes and acoustic modes suggests that the energy transfer between those modes is very likely and easy. As a result, the softening optic modes must have a strong scattering effect on the heat-carrying acoustic modes, which may be the mechanism of the ultralow lattice thermal conductivity in chalcostibite.

Fig. 11 shows the calculated total and partial phonon density of states (DOS) of (a) $\mathrm{CuSbS}_{2}$ and (b) $\mathrm{CuGaS}_{2}$. The peaks of the DOS shift toward higher frequency in $\mathrm{CuGaS}_{2}$ because of the lower mass of Ga compared with Sb. In fact, most of the modes with frequency higher than $160 \mathrm{~cm}^{-1}$ in $\mathrm{CuSbS}_{2}(275$ $\mathrm{cm}^{-1}$ in $\mathrm{CuGaS}_{2}$ ) are only connected to the $\mathrm{S}$ and $\mathrm{Cu}$ bonds, while the $\mathrm{Sb}$ bonds contribute to the peaks below $153 \mathrm{~cm}^{-1}$ in chalcostibite (Ga below $236 \mathrm{~cm}^{-1}$ in $\mathrm{CuGaS}_{2}$ ). To elaborate the difference between the two materials, the details of the phonon DOS between 20 and $150 \mathrm{~cm}^{-1}$ are highlighted in Fig. 11(c) and (d). In $\mathrm{CuGaS}_{2}$, there is only one Ga-related broad peak at around $90 \mathrm{~cm}^{-1}$. There are no significant contributions from Ga out of the range of $70-105 \mathrm{~cm}^{-1}$. In sharp contrast to $\mathrm{CuGaS}_{2}$, there are three apparent Sb related peaks at 82, 111 and $138 \mathrm{~cm}^{-1}$ for $\mathrm{CuSbS}_{2}$. Except for the peak at around 111 $\mathrm{cm}^{-1}$ showing a broad shoulder similar to that observed in $\mathrm{Ga}$ substituted $\mathrm{CuGaS}_{2}$, the other two peaks are very sharp and show nearly no shoulders on the high frequency side. This is the characteristic of quasi-single-frequency Einstein modes created by the individual 'rattling' of the guest atoms in skutterudites and clathrates. ${ }^{21,72}$ In $\mathrm{CuSbS}_{2}$, the phonon structure is well defined and can be understood in terms of the ideal crystal. No glass-like behaviour was observed. However, the two Sb-related quasi-single-frequency vibration modes behave like localised Einstein harmonic oscillators and qualitatively modified the whole vibration spectrum. ${ }^{73}$ These results give unequivocal evidence that the trivalent $\mathrm{V}_{\mathrm{A}}$ atom creates special bonding and vibration modes because of its nonbonding $5 \mathrm{~s}$ lone-pair electrons. Combined with the crystal structure shown in Fig. 2(d), we conjecture that quasi-single-frequency modes may be related to the $\mathrm{Sb}$ vibration in a direction perpendicular to the $\mathrm{SbS}_{4}$ plane of the pyramidal $\mathrm{SbS}_{5}$ unit. Due to the asymmetric bonding, $\mathrm{Sb}$ atoms are free to undergo large displacement or shift toward the voids separating the $\mathrm{SbS}_{5}$ units, while the movement toward the vertex is restrained by the opposing $\mathrm{S}$ atoms. Moreover, the non-bonding $5 \mathrm{~s}^{2}$ electrons are expected to form a shell of relatively large radius, especially in the direction toward the voids. During the thermal vibration, the wave functions of lone-pair electrons overlap with each other, which leads to an additional repulsive force to the restoring force that in turn leads to extreme anharmonicity of the lattice vibrational spectrum. ${ }^{34}$ This is likely to be the origin of the ultralow lattice thermal conductivity in systems with lone-pair electrons.
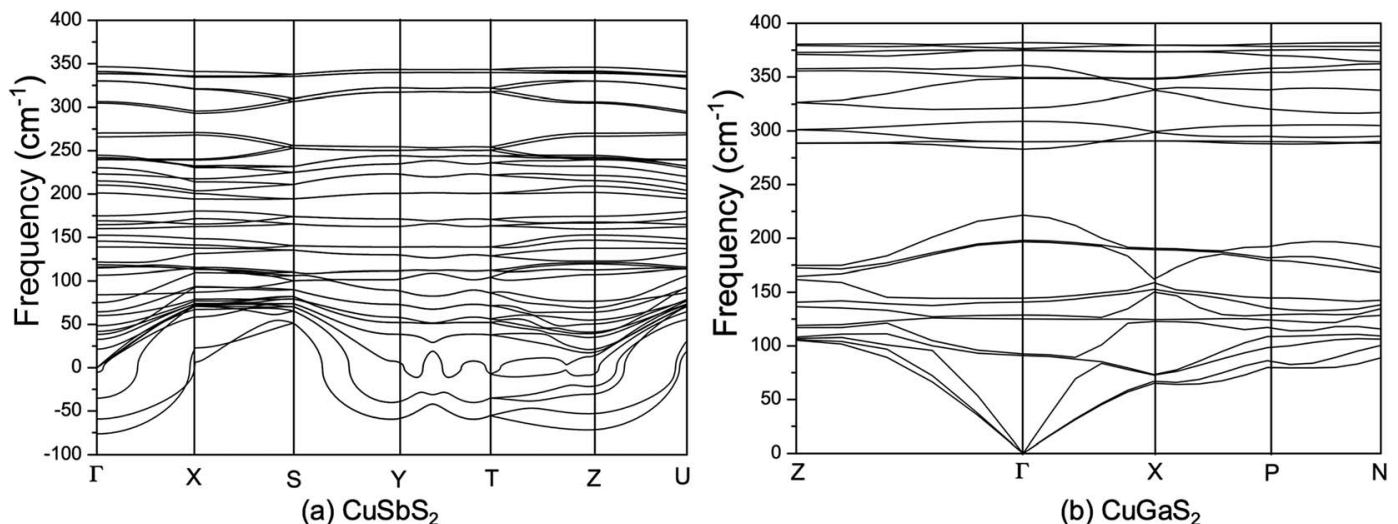

(b) $\mathrm{CuGaS}_{2}$

Fig. 10 The phonon dispersions of (a) $\mathrm{CuSbS}_{2}$ and (d) $\mathrm{CuGaS}_{2}$ compounds. 

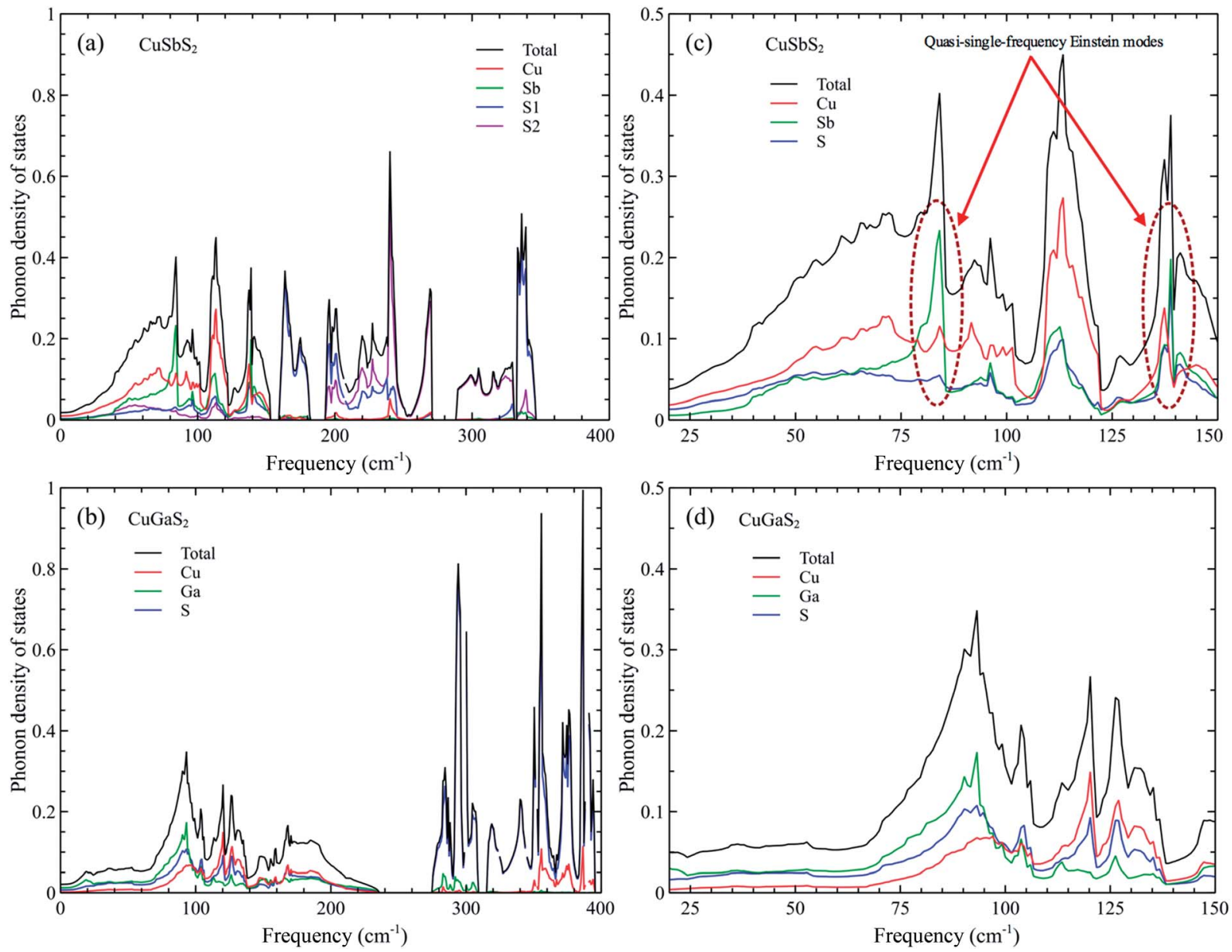

Fig. 11 Calculated total and partial phonon density of states (DOS) with appropriate scaling of (a, c) CuSbS 2 and (b, d) CuGaS 2 . Two quasi-singlefrequency Einstein modes created by lone-pair electrons on Sb site are labelled in (c).

\section{Conclusions}

To clarify the importance of lone-pair electrons on trivalent $\mathrm{Sb}^{3+}$ sites in $\mathrm{Cu}-\mathrm{Sb}-\mathrm{S}$ systems, we purposely introduced alloy phonon scattering in the carefully chosen chalcostibite $\mathrm{CuSbS}_{2}$ and used it as a benchmark to quantify the impact of lone-pair electrons on thermal transport properties. The thermal conductivity measurements show an apparent deviation from the conventional alloy model. Most impressively, the role of lone-pair electrons eclipses point defects in lightly doped solid solutions, which gives the first direct demonstration of the importance of the lone-pair electrons by comparison with another well-known scattering mechanism. Phonon dispersion calculations disclosed two $\mathrm{Sb}$ related quasi-single-frequency vibration modes behaving like localised Einstein harmonic oscillators, similar to the modes created by the individual 'rattling' of the guest atoms in skutterudites and clathrates. Combined with the crystal structure evolution with increasing substitution, we conjecture that quasi-single-frequency modes may be related to the $\mathrm{Sb}$ vibration in a direction perpendicular to the $\mathrm{SbS}_{4}$ plane of the pyramidal $\mathrm{SbS}_{5}$ unit. Due to the asymmetric bonding, the wave functions of lone-pair electrons in the voids overlap with each other during thermal vibration, which leads to an additional repulsive force on the restoring force, which leads to extreme anharmonicity of the lattice vibrational spectrum and ultralow lattice thermal conductivity in chalcostibite. This suggests that compounds with lone-pair electrons (trivalent $\mathrm{V}_{\mathrm{A}}$ atoms) are a new direction to design low thermal conductivity materials.

\section{Acknowledgements}

This work was supported by a Marie Curie International Incoming Fellowship of the European Community Human Potential Program under Contract no. PIIF-GA-2013-622847. RZ acknowledges the support from a Marie Curie International Incoming Fellowship (contract number, PIIF-GA-2013-624474). KC acknowledges support from China Scholarship Council (CSC). MJR and AM would also like to acknowledge the support of The Engineering and Physical Sciences Research Council (EP/ L017695/1, MASSIVE). BD also thanks the financial supports of the National Science Foundation of China (Grant No. 51202060).

\section{References}

1 Handbook of Thermoelectrics: Macro to Nano, ed. D. M. Rowe, CRC press/Taylor \& Francis, Boca Raton, FL, 2006. 
2 E. S. Toberer, A. Zevalkink and G. J. Snyder, J. Mater. Chem., 2011, 21, 15843-15852.

3 G. A. Slack, New materials and performance limits for the thermoelectric cooling, in CRC handbook of thermoelectrics, CRC Press, Boca Raton, FL, 1995.

4 A. J. Minnich, M. S. Dresselhaus, Z. F. Ren and G. Chen, Energy Environ. Sci., 2009, 2, 466-479.

5 B. Qiu, Z. Tian, A. Vallabhaneni, B. Liao, J. M. Mendoza, O. D. Restrepo, X. Ruan and G. Chen, Europhys. Lett., 2015, 109, 57006.

6 K. F. Hsu, S. Loo, F. Guo, W. Chen, J. S. Dyck, C. Uher, T. Hogan, E. K. Polychroniadis and M. G. Kanatzidis, Science, 2004, 303, 818-821.

7 W. Xie, J. He, H. J. Kang, X. Tang, S. Zhu, M. Laver, S. Wang, J. R. D. Copley, C. M. Brown, Q. Zhang and T. M. Tritt, Nano Lett., 2010, 10, 3283-3289.

8 B. Poudel, Q. Hao, Y. Ma, Y. Lan, A. Minnich, B. Yu, X. Yan, D. Wang, A. Muto, D. Vashaee, X. Chen, J. Liu, M. S. Dresselhaus, G. Chen and Z. Ren, Science, 2008, 320, 634-638.

9 B. Du, H. Li, J. Xu, X. Tang and C. Uher, J. Solid State Chem., 2011, 184, 109-114.

10 A. U. Khan, K. Kobayashi, D.-M. Tang, Y. Yamauchi, K. Hasegawa, M. Mitome, Y. Xue, B. Jiang, K. Tsuchiya, D. Golberg, Y. Bando and T. Mori, Nano Energy, 2017, 31, 152-159.

11 X. Tang, W. Xie, H. Li, B. Du, Q. Zhang, T. M. Tritt and C. Uher, High-Performance Nanostructured Thermoelectric Materials Prepared by Melt Spinning and Spark Plasma Sintering, in Materials, Preparation, and Characterization, in Thermoelectrics, D.M. Rowe, ed. CRC press, Boca Raton, FL, 2012.

12 G. J. Snyder and E. S. Toberer, Nat. Mater., 2008, 7, 105114.

13 G. A. Slack, Crystal complexity, in Semiconductors and Semimetals, Academic Press, New York, 34th edn, 1979.

14 T. Mori, J. Martin and G. Nolas, J. Appl. Phys., 2007, 102, 73510.

15 K. Koumoto, I. Terasaki and R. Funahashi, MRS Bull., 2006, 31, 206-210.

16 C. Wan, X. Gu, F. Dang, T. Itoh, Y. Wang, H. Sasaki, M. Kondo, K. Koga, K. Yabuki, G. J. Snyder, R. Yang and K. Koumoto, Nat. Mater., 2015, 14, 622-627.

17 P. Vaqueiro, G. Guelou, M. Stec, E. Guilmeau and A. V. Powell, J. Mater. Chem. A, 2013, 1, 520-523.

18 X. Shi, W. Zhang, L. D. Chen and J. Yang, Phys. Rev. Lett., 2005, 95, 185503.

19 X. Shi, W. Zhang, L. D. Chen, J. Yang and C. Uher, Acta Mater., 2008, 56, 1733-1740.

20 M. Christensen, A. B. Abrahamsen, N. B. Christensen, F. Juranyi, N. H. Andersen, K. Lefmann, J. Andreasson, C. R. H. Bahl and B. B. Iversen, Nat. Mater., 2008, 7, 811-815.

21 T. Tadano, Y. Gohda and S. Tsuneyuki, Impact of Rattlers on Thermal Conductivity of a Thermoelectric Clathrate: A FirstPrinciples Study. PG-095501. Department of Physics, The University of Tokyo, Tokyo 113-0033, Japan, FAU-Gohda, Yoshihiro.
22 X. Shi, J. Yang, J. R. Salvador, M. Chi, J. Y. Cho, H. Wang, S. Bai, J. Yang, W. Zhang and L. Chen, J. Am. Chem. Soc., 2011, 133, 7837-7846.

23 T. Takabatake, K. Suekuni and T. Nakayama, Rev. Mod. Phys., 2014, 86, 669-716.

24 C. W. Li, J. Hong, A. F. May, D. Bansal, S. Chi, T. Hong, G. Ehlers and O. Delaire, Nat. Phys., 2015, 11, 1063-1069.

25 L.-D. Zhao, S.-H. Lo, Y. Zhang, H. Sun, G. Tan, C. Uher, C. Wolverton, V. P. Dravid and M. G. Kanatzidis, Nature, 2014, 508, 373-377.

26 G. F. HändelSuite No. 5 in E major, HWV 430, 1720.

27 J. P. Heremans, Nat. Phys., 2015, 11, 990-991.

28 H. Liu, X. Shi, F. Xu, L. Zhang, W. Zhang, L. Chen, Q. Li, C. Uher, T. Day and G. J. Snyder, Nat. Mater., 2012, 11, 422-425.

29 L. Zhao, X. Wang, J. Wang, Z. Cheng, S. Dou, J. Wang and L. Liu, Sci. Rep., 2015, 5, 7671.

30 E. J. Skoug and D. T. Morelli, Phys. Rev. Lett., 2011, 107, 235901.

31 D. T. Do and S. D. Mahanti, J. Phys. Chem. Solids, 2014, 75, 477-485.

32 D. Do, V. Ozolins, S. D. Mahanti, M.-S. Lee, Y. Zhang and C. Wolverton, J. Phys.: Condens. Matter, 2012, 24, 415502.

33 K. Tyagi, B. Gahtori, S. Bathula, A. K. Srivastava, A. K. Shukla, S. Auluck and A. Dhar, J. Mater. Chem. A, 2014, 2, 1582915835.

34 D. T. Morelli, V. Jovovic and J. P. Heremans, Phys. Rev. Lett., 2008, 101, 35901.

35 V. Jovovic and J. P. Heremans, Phys. Rev. B: Condens. Matter Mater. Phys., 2008, 77, 245204.

36 L.-H. Ye, K. Hoang, A. J. Freeman, S. D. Mahanti, J. He, T. M. Tritt and M. G. Kanatzidis, Phys. Rev. B: Condens. Matter Mater. Phys., 2008, 77, 245203.

37 W. Kim, J. Mater. Chem. C, 2015, 3, 10336-10348.

38 U. V. Waghmare, N. A. Spaldin, H. C. Kandpal and R. Seshadri, Phys. Rev. B: Condens. Matter Mater. Phys., 2003, 67, 125111.

39 K. Ramasamy, H. Sims, W. H. Butler and A. Gupta, Chem. Mater., 2014, 26, 2891-2899.

40 D. T. Do and S. D. D. Mahanti, J. Phys. Chem. Solids, 2014, 75, 477-485.

41 B. Du, K. Chen, H. Yan and M. J. Reece, Scr. Mater., 2016, 111, 49-53.

42 B. Du, H. Li, J. Xu, X. Tang and C. Uher, Chem. Mater., 2010, 22, 5521-5527.

43 J. Xu, H. Li, B. Du, X. Tang, Q. Zhang and C. Uher, J. Mater. Chem., 2010, 20, 6138-6143.

44 J. Ma, O. Delaire, A. F. May, C. E. Carlton, M. A. McGuire, L. H. VanBebber, D. L. Abernathy, G. Ehlers, T. Hong, A. Huq, W. Tian, V. M. Keppens, Y. Shao-Horn and B. C. Sales, Nat. Nanotechnol., 2013, 8, 445-451.

45 M. D. Nielsen, V. Ozolins and J. P. Heremans, Energy Environ. Sci., 2013, 6, 570-578.

46 S. Kumar and U. Schwingenschlogl, Phys. Chem. Chem. Phys., 2016, 18, 19158-19164. 
47 P. Vaqueiro, R. A. R. Al Orabi, S. D. N. Luu, G. Guelou, A. V. Powell, R. I. Smith, J.-P. Song, D. Wee and M. Fornari, Phys. Chem. Chem. Phys., 2015, 17, 31735-31740.

48 A. Suzumura, M. Watanabe, N. Nagasako and R. Asahi, J. Electron. Mater., 2014, 43, 2356-2361.

49 Y. Goto, Y. Sakai, Y. Kamihara and M. Matoba, J. Phys. Soc. Jpn., 2015, 84, 44706.

50 J. Heo, G. Laurita, S. Muir, M. A. Subramanian and D. A. Keszler, Chem. Mater., 2014, 26, 2047-2051.

51 X. Lu and D. T. Morelli, Phys. Chem. Chem. Phys., 2013, 15, 5762-5766.

52 X. Lu, D. T. Morelli, Y. Xia, F. Zhou, V. Ozolins, H. Chi, X. Zhou and C. Uher, Adv. Energy Mater., 2013, 3, 342-348.

53 H. J. Whitfield, Solid State Commun., 1980, 33, 747-748.

54 K. Ramasamy, H. Sims, W. H. Butler and A. Gupta, J. Am. Chem. Soc., 2014, 136, 1587-1598.

55 A. F. Ioffe, Semiconductor thermoelements, and Thermoelectric cooling, Infosearch, ltd., 1957.

56 V. Kumar Gudelli, V. Kanchana, G. Vaitheeswaran, A. Svane and N. E. Christensen, J. Appl. Phys., 2013, 114, 223707.

57 K. Chen, B. Du, N. Bonini, C. Weber, H. Yan and M. J. Reece, J. Phys. Chem. C, 2016, 120, 27135-27140.

58 Famatinite $\left(\mathrm{Cu}_{3} \mathrm{SbS}_{4}\right)$ Crystal Structure, Lattice Parameters, Physical Properties. Datasheet from Landolt-Börnstein - Group III Condensed Matter: 'Ternary Compounds, Organic Semiconductors' in SpringerMaterials, ed.O. Madelung, U. Rössler and M. Schulz, Springer-Verlag, Berlin, Heidelberg, 2000. vol. 41E.

59 W. Lai, Y. Wang, D. T. Morelli and X. Lu, Adv. Funct. Mater., 2015, 25, 3648-3657.
60 J. T. R. Dufton, A. Walsh, P. M. Panchmatia, L. M. Peter, D. Colombara and M. S. Islam, Phys. Chem. Chem. Phys., 2012, 14, 7229-7233.

61 P. Giannozzi, S. Baroni, N. Bonini, M. Calandra, R. Car, C. Cavazzoni, D. Ceresoli, G. L. Chiarotti, M. Cococcioni, I. Dabo1, A. Dal Corso, S. G. De and S. F. Wentzcovitch, J. Phys.: Condens. Matter, 2009, 21, 395502.

62 K. F. Garrity, J. W. Bennett, K. M. Rabe and D. Vanderbilt, Comput. Mater. Sci., 2014, 81, 446-452.

63 J. Baker, R. S. Kumar, D. Sneed, A. Connolly, Y. Zhang, N. Velisavljevic, J. Paladugu, M. Pravica, C. Chen, A. Cornelius and Y. Zhao, J. Alloys Compd., 2015, 643, 186194.

64 G. V. Chester and A. Thellung, Proc. Phys. Soc., 1961, 77, 1005.

65 D. Berthebaud, O. I. Lebedev and A. Maignan, J. Mater., 2015, 1, 68-74.

66 N. Tsujii, T. Mori and Y. Isoda, J. Electron. Mater., 2014, 43, 2371-2375.

67 P. W. Yu, D. L. Downing and Y. S. Park, J. Appl. Phys., 1974, 45, 5283-5288.

$68 \mathrm{~S}$. Scherrer and H. Scherrer, in CRC Handbook of Thermoelectrics, ed. D.M. Rowe, CRC Press, Boca Raton, FL, 1995.

69 C. Vining, in CRC Handbook of Thermoelectrics, ed. D. M. Rowe, CRC Press, Boca Raton, FL, 1995.

70 B. Abeles, Phys. Rev., 1963, 131, 1906-1911.

71 P. G. Klemens, Phys. Rev., 1960, 119, 507-509.

72 B. C. Sales, D. Mandrus and R. K. Williams, Science, 1996, 272, 1325-1328.

73 R. P. Hermann, F. Grandjean and G. J. Long, Am. J. Phys., 2005, 73, 110-118. 